\title{
Is Neoadjuvant Therapy Needed in Patients with Resectable Pancreatic Cancer?
}

\author{
Dong Uk Kim \\ Department of Internal Medicine, Pusan National University College of Medicine and Institute, Pusan National University Hospital, \\ Busan, Korea
}

\author{
Corresponding Author \\ Dong Uk Kim \\ ORCID https://orcid.org/0000-0002-7208-7753 \\ E-mail amlm3@hanmail.net
}

\begin{abstract}
See "Response to Neoadjuvant Therapy and Prognosis in Patients with Resectable Pancreatic Cancer: A Propensity Score Matching Analysis" by Min Sung Yoon, et al. on page
\end{abstract} 118, Vol. 16, No. 1, 2022
Pancreatic cancer is representative cancer with a poor prognosis. Surgery is the only way to expect a cure, but most of the patients are presented in inoperable, advanced disease. Furthermore, most pancreatic cancer patients eventually experience local or distant recurrence even after complete surgical resection, resulting in 5-year survival of $12 \%$ to $27 \%$. $^{1,2}$ In order to improve surgical outcomes, neoadjuvant chemotherapy or chemoradiation therapy is often performed in patients with borderline resectable pancreatic cancer. In a meta-analysis of 38 studies, ${ }^{3}$ patients with borderline resectable tumors, who underwent neoadjuvant therapy, showed longer median survival compared to those who underwent upfront surgery (19.2 months vs 12.8 months, respectively). R0 resection rate in neoadjuvant therapy group also increased to $88.6 \%$ compared to $63.9 \%$ in upfront surgery group. Pancreatic cancer is considered to be locally invasive and systemically spread even in patients with no evidence of metastasis on imaging. Therefore, neoadjuvant chemotherapy or chemoradiation therapy may be required even in patients with tumors that can be anatomically resected.

Yoon et al. ${ }^{4}$ reviewed 202 patients with resectable pancreatic cancer. One hundred sixty-seven patients underwent surgery immediately, and 35 patients received neoadjuvant chemotherapy or chemoradiation therapy. For 35 patients who underwent neoadjuvant therapy, matching was performed with patients who underwent upfront surgery at a ratio of 1:3. In neoadjuvant therapy group, the R0 resection was $74.3 \%$, which was significantly higher than that of $51.5 \%$ in upfront surgery group. The progression- free survival (PFS) was 29.6 months, which was significantly higher than that of the patients who underwent surgery first at 15.1 months. However, there was no difference in overall survival between the two groups. The authors assumed that neoadjuvant therapy in patients with resectable disease could prolong PFS although selection bias still exists because this is a retrospective study.

The standard treatment for resectable pancreatic cancer is to perform chemotherapy after surgery. Recently, FOLFIRINOX (irinotecan, oxaliplatin, 5-fluorouracil, and leucovorin) after surgery showed a dramatically increased overall survival of 54.4 months compared to 35 months of gemcitabine monotherapy. ${ }^{5}$ Despite the improvement in survival period, some patients still experience recurrence, and the need for chemotherapy or chemoradiation therapy before surgery is emerging.

In the retrospective review from the National Cancer Database from 2006 to 2012, 15,237 patients with clinical stage I or II resected pancreatic cancer were identified and 2,005 patients from the neoadjuvant therapy group were matched with 6,015 patients who underwent upfront surgery. ${ }^{6}$ The neoadjuvant therapy group showed longer survival compared to that of upfront surgery (26 months vs 21 months, respectively; $\mathrm{p}<0.01$ ).

In PREOPANC trial, ${ }^{7}$ a randomized phase III study of chemoradiation therapy versus immediate surgery in patients with resectable or borderline resectable disease, median overall survival was 16.0 months with neoadjuvant chemoradiation therapy and 14.3 months with upfront surgery (hazard ratio, $0.78 ; 95 \%$ confidence interval, 0.58 
to $1.05 ; \mathrm{p}=0.096$ ). The $\mathrm{R} 0$ resection rate was $71 \%$ (51 of 72 ) in patients who received neoadjuvant chemoradiation therapy and 40\% (37 of 92) in patients assigned to upfront surgery $(p<0.001)$. Furthermore, a study to see the usefulness of FOLFIRINOX in the PREOPANC-2 study is ongoing. ${ }^{8}$ Total neoadjuvant FOLFIRINOX versus neoadjuvant gemcitabine-based chemoradiation therapy and adjuvant gemcitabine chemotherapy will be compared.

In SWOG S1505, ${ }^{9}$ a randomized phase II study of patients with resectable disease, which aimed at determining the most promising perioperative regimen, 55 patients were treated with mFOLFIRINOX and 47 patients with gemcitabine/nab-paclitaxel. There was no difference in survival or surgical outcome between the two groups.

I assume that neoadjuvant therapy is a favorable trend for biologically borderline resectable disease in patients with anatomically resectable status. ${ }^{10}$ Pancreatic cancer may be already micro-metastatic disease even in anatomically resectable status. Therefore, I wonder which factors are significant to figure out the biological activity of tumor in anatomically resectable pancreatic cancer. National Comprehensive Cancer Network guideline suggests neoadjuvant therapy in high-risk pancreatic ductal adenocarcinoma patients. High-risk features include high carbohydrate antigen 19-9, large primary tumor size, large regional lymph nodes, excessive weight loss, and extreme pain.

There are many unresolved problems with neoadjuvant therapy for resectable pancreatic cancer. Furthermore, we need a diagnostic method that can biologically confirm whether a tumor can be resected and whether there is no micrometastasis. Regarding neoadjuvant therapy, more research is needed particularly on the appropriate regimen and duration.

\section{CONFLICTS OF INTEREST}

No potential conflict of interest relevant to this article was reported.

\section{ORCID}

Dong Uk Kim https://orcid.org/0000-0002-7208-7753

\section{REFERENCES}

1. Ryan DP, Hong TS, Bardeesy N. Pancreatic adenocarcinoma. N Engl J Med 2014;371:1039-1049.

2. Groot VP, Rezaee N, Wu W, et al. Patterns, timing, and predictors of recurrence following pancreatectomy for pancreatic ductal adenocarcinoma. Ann Surg 2018;267:936-945.

3. Versteijne E, Vogel JA, Besselink MG, et al. Meta-analysis comparing upfront surgery with neoadjuvant treatment in patients with resectable or borderline resectable pancreatic cancer. Br J Surg 2018;105:946-958.

4. Yoon MS, Lee HS, Kang CM, et al. Response to neoadjuvant therapy and prognosis in patients with resectable pancreatic cancer: a propensity score matching analysis. Gut Liver 2022;16:118-128.

5. Conroy T, Hammel P, Hebbar M, et al. FOLFIRINOX or gemcitabine as adjuvant therapy for pancreatic cancer. $\mathrm{N}$ Engl J Med 2018;379:2395-2406.

6. Mokdad AA, Minter RM, Zhu H, et al. Neoadjuvant therapy followed by resection versus upfront resection for resectable pancreatic cancer: a propensity score matched analysis. J Clin Oncol 2017;35:515-522.

7. Versteijne E, Suker M, Groothuis K, et al. Preoperative chemoradiotherapy versus immediate surgery for resectable and borderline resectable pancreatic cancer: results of the Dutch randomized phase III PREOPANC trial. J Clin Oncol 2020;38:1763-1773.

8. Janssen QP, van Dam JL, Bonsing BA, et al. Total neoadjuvant FOLFIRINOX versus neoadjuvant gemcitabine-based chemoradiotherapy and adjuvant gemcitabine for resectable and borderline resectable pancreatic cancer (PREOPANC-2 trial): study protocol for a nationwide multicenter randomized controlled trial. BMC Cancer 2021;21:300.

9. Sohal DPS, Duong M, Ahmad SA, et al. Efficacy of perioperative chemotherapy for resectable pancreatic adenocarcinoma: a phase 2 randomized clinical trial. JAMA Oncol 2021;7:421-427.

10. Isaji S, Mizuno S, Windsor JA, et al. International consensus on definition and criteria of borderline resectable pancreatic ductal adenocarcinoma 2017. Pancreatology 2018;18:2-11. 